with and without other autoantibodies present using 451 SSc patients with complete dataset. Autoantibodies to several scleroderma-related autoantibodies were detected using commercial or research use only kits (Inova Diagnostics, San Diego, USA). P-values below 0.05 were considered significant.

Results: The sensitivity and specificity of anti-BICD2 antibodies were determined as $22.1 \%$ and $99.0 \%$, respectively. Receiver operating characteristic (ROC) analysis showed an area under the curve of $0.79(95 \% \mathrm{Cl} 0.75-0.84)$. The likelihood (LR) and odds ratios (OR) were 17.7 (LR+), 0.8 (LR-) and 20.4 (OR). The prevalence of these autoantibodies was equally distributed between limited and diffuse form of SSc $(22.8 \%$ and $24.0 \%)$. When SSc patients without the classification criteria markers anti-Scl-70, anti-Centromere and anti-RNA Pol III $(n=184)$ were compared with controls, similar performance was obtained as seen in the entire cohort. Results are summarized in table 1 below.

\begin{tabular}{lcc} 
Table 1 & \\
\hline Performance Characteristic & $\begin{array}{c}\text { Entire SSc cohort } \\
(\mathrm{n}=502)\end{array}$ & $\begin{array}{c}\text { Anti-Scl-70, anti-Centromere } \\
\text { and anti-RNA Pol III negative }(\mathrm{n}=184)\end{array}$ \\
\hline Sensitivity & $22.1 \%$ & $13.6 \%$ \\
Specificity & $99.0 \%$ & $99.0 \%$ \\
AUC & $0.79(95 \% \mathrm{Cl} 0.75-0.84)$ & $0.78(95 \% \mathrm{Cl} 0.72-0.84)$ \\
LR+ & 28.1 & 15.6 \\
LR- & 0.21 & 0.13 \\
OR & 133.1 & 123.7
\end{tabular}

Several statistically relevant clinical associations were found for anti-BICD2 as summarized in table 2 below.

Conclusions: Our data confirm the presence of anti-BICD2 antibodies in SSC patients that may help to differentiate SSc from other systemic autoimmune rheumatic diseases and to stratify SSc patients into more defined subforms of the disease.

Disclosure of Interest: M. Mahler Employee of: Inova Diagnostics, C. Bentow Employee of: Inova Diagnostics, J. Milo Employee of: Inova Diagnostics, M. Hudson: None declared, H.-D. Zucht Employee of: Protagen, P. Budde Employee of: Protagen, D. Wirtz Employee of: Protagen, J. Schulte-Pelkum Employee of: Protagen, M. Choi: None declared, M. Fritzler: None declared DOI: 10.1136/annrheumdis-2017-eular.2751

\section{SAT0373 EVALUATION OF AMERICAN COLLEGE OF RHEUMATOLOGY PROVISIONAL COMPOSITE RESPONSE (CRISS) INDEX IN THE FASSCINATE TRIAL}

D. Khanna ${ }^{1}$, V. Berrocal ${ }^{1}$, C. Denton ${ }^{2}$, A. Jaheris ${ }^{3}$, H. Spotwood ${ }^{4}$, C. Lin $^{3}$, J. Siegel ${ }^{3}$, D. Furst ${ }^{5} .{ }^{1}$ Univ Michigan, Ann Arbor, United States; ${ }^{2}$ UCL, London, United Kingdom; ${ }^{3}$ Genentech, San Francisco, United States; ${ }^{4}$ Roche, Welwyn Garden, United Kingdom; ${ }^{5}$ UCLA, Los Angeles, United States

Background: Treatment with tocilizumab (TCZ) in early systemic sclerosis (SSc; FaSScinate trial) resulted in consistent, but not statistically significant, improvements in skin sclerosis (mRSS) at wks 24 and $48^{1}$. The CRISS index has been proposed as a composite index for trials in SSc ${ }^{2}$. CRISS is a 2-step process that assigns a probability of improvement for each subject ranging from 0.0 [no improvement] to 1.0 [marked improvement]. Step 1 assesses clinically meaningful decline in cardio-pulmonary-renal involvement and assigns a probability of 0.0 . For remaining subjects, 5 variables are used to calculate probability of improvement. These are: FVC\%, mRSS, patient (PT GA) and physician globals (MD GA), and HAQ-DI.

Objectives: To assess the performance of CRISS index in the FaSScinate trial. Methods: In FaSScinate, pts $\geq 18$ y with active SSc were randomized 1:1 to TCZ or placebo (PLA) for 48 wks. Step 1 CRISS was captured using review of serious adverse event data. Non-parametric Wilcoxon test was used to assess significant differences between the CRISS scores in both arms. Analyses were carried out for subject who had complete data at baseline and 24 weeks and at baseline and 48 weeks.

Results: 87 pts (43 TCZ, 44 PBO) were enrolled. Baseline characteristics were similar between arms. 4 subjects in the PBO group and none in TCZ met the pre-defined definition of worsening in Step 1 and were given a score of 0.0 . For remaining subjects, we calculated probability score for each subject. Using CRISS as a continuous measure, the median score was statistically significant and favored TCZ compared to PBO at wks 24 and 48 ( $\mathrm{p}=0.04$ and 0.01 ; Table). Table also presents the individual components of Step 2 where only FVC\% was statistically significant favoring TCZ at wk 48 .
Table 1. Comparison of TCZ and PBO using CRISS index and individual variables at 24 and 48 weeks

\begin{tabular}{lccc}
\hline & $\mathrm{TCZ}$ & $\mathrm{PBO}^{* *}$ & $\mathrm{P}$ value \\
\hline CRISS, median [IQR] at 24 week & 0.23 & 0.008 & $0.04^{*}$ \\
& {$[0.003 ; 0.95], \mathrm{N}=33$} & {$[0.00 ; 0.10], \mathrm{N}=36$} & \\
CRISS, median [IQR] at 48 week & 0.31 & 0.001 & $0.01^{*}$ \\
& {$[0.008 ; 0.99], \mathrm{N}=27$} & {$[0.00 ; 0.18], \mathrm{N}=35$} & \\
MRSS, mean change at 24 week & $-4.2, \mathrm{~N}=33$ & $-2.1, \mathrm{~N}=34$ & 0.24 \\
MRSS, mean change at 48 week & $-5.9, \mathrm{~N}=27$ & $-3.2, \mathrm{~N}=31$ & 0.35 \\
FVC\% predicted, mean change at 24 week & $-0.78, \mathrm{~N}=33$ & $-4.4, \mathrm{~N}=34$ & 0.08 \\
FVC\% predicted, mean change at 48 week & $-1.6, \mathrm{~N}=27$ & $-5.1, \mathrm{~N}=31$ & 0.02 \\
PT GA, mean change at 24 week & $-0.41, \mathrm{~N}=33$ & $-0.10, \mathrm{~N}=34$ & 0.64 \\
PT GA, mean change at 48 week & $-0.69, \mathrm{~N}=27$ & $-0.45, \mathrm{~N}=31$ & 0.72 \\
MD GA, mean change at 24 week & $-0.94, \mathrm{~N}=33$ & $-0.80, \mathrm{~N}=34$ & 0.76 \\
MDGA, mean change at 48 week & $-1.81, \mathrm{~N}=27$ & $-0.73, \mathrm{~N}=31$ & 0.07 \\
HAQ-DI, mean change at 24 week & $0.09, \mathrm{~N}=33$ & $0.06, \mathrm{~N}=34$ & 0.76 \\
HAQ-DI, mean change at 48 week & $0.00, \mathrm{~N}=27$ & $0.16, \mathrm{~N}=31$ & 0.10 \\
\hline
\end{tabular}

*Using Wilcoxon test as CRISS data is not normally distributed. ${ }^{* \star} T h e r e$ are 4 subjects in the PBO who met step 1 and were given a score of 0.0 .

Conclusions: In this post-hoc analysis, CRISS index was able to discriminate TCZ from PBO, supporting its validity in an independent cohort.

References:

[1] Khanna D. Lancet. 2016

[2] Khanna D. Arthritis Rheum. 2016.

Disclosure of Interest: D. Khanna Grant/research support from: NIH/NIAMS and NIH/NIAID, BMS, Pfizer, Consultant for: Actelion, Bayer, BMS, Boehringer Ingelheim, Genentech/Roche, Sanofi-aventis, GSK, Corbus, Cytori, EMD Serono, V. Berrocal: None declared, C. Denton Consultant for: Actelion, Bayer, GSK, CSL Behring, Merck-Serono, Genentech-Roche, Inventiva, Sanofi-Aventis, Boehringer Ingelheim, A. Jaheris Shareholder of: Roche stock and options, Employee of: Genentech, H. Spotwood Employee of: Roche, C. Lin Employee of: Genentech, J. Siegel Employee of: Genentech, D. Furst Grant/research support from: Amgen, BMS, NIH, Novartis, Pfizer, Roche/Genentech, Consultant for: AbbVie, Amgen, BMS, Cytori, Novartis, Pfizer, Roche/Genentech, UCB

DOI: 10.1136/annrheumdis-2017-eular.3754

\section{SAT0374 THE EUROMYOSITIS REGISTRY: AN INTERNATIONAL DESCRIPTION OF MYOSITIS}

J.B. Lilleker ${ }^{1}$, J. Vencovsky ${ }^{2}$, G. Wang ${ }^{3}$, L.R. Wedderburn ${ }^{4}$, L.P. Diederichsen ${ }^{5}$, J. Schmidt ${ }^{6}$, P. Jordan ${ }^{7}$, O. Benveniste ${ }^{8}$, M.G. Danieli ${ }^{9}$, K. Dankó $^{10}$, N.T.P. Thuy ${ }^{11}$, M. Vazquez-Del Mercado ${ }^{12}, \varnothing$. Molberg ${ }^{13}$, B. De Paepe ${ }^{14}$, J. De Bleecker $^{15}$, B. Maurer ${ }^{16}$, N. Pipitone ${ }^{17}$, N. McHugh ${ }^{18,19}$, Z. Betteridge ${ }^{18,19}$, P. New ${ }^{20}$, R.G. Cooper ${ }^{20}$, W.E. Ollier ${ }^{1}$, J.A. Lamb ${ }^{1}$, N.S. Krogh ${ }^{21}$,

I.E. Lundberg ${ }^{22}, \mathrm{H}$. Chinoy ${ }^{23}$ on behalf of UKMYONET and all contributors to the EuroMyositis Registry. ${ }^{1}$ Faculty of Biology, Medicine and Health, The University of Manchester, Manchester, United Kingdom; ${ }^{2}$ Institute of Rheumatology, Charles University, Prague, Czech Republic; ${ }^{3}$ Rheumatology, China-Japan Friendship Hospital, Beijing, China; ${ }^{4}$ UCL GOS Institute of Child Health, London, United Kingdom; ${ }^{5}$ Rheumatology, Odense University Hospital, Odense, Denmark; ${ }^{6}$ Neurology, University Medical Center, Göttingen, Germany; ${ }^{7}$ Myositis UK, Southampton, United Kingdom; ${ }^{8}$ Médecine Interne et Immunologie Clinique, Hôpital Pitié-Salpêtrière, Paris, France; ${ }^{9}$ Scienze Cliniche e Molecolari,

Università Politecnica delle Marche \& Ospedali Riuniti, Ancona, Italy;

${ }^{10}$ Immunology, University of Debrecen, Debrecen, Hungary; ${ }^{11}$ Rheumatology,

Bach Mai Hospital, Hanoi, Viet Nam; ${ }^{12}$ Medicina Interna, Hospital Civil Dr. Juan I. Menchaca, Guadalajara, Mexico: ${ }^{13}$ Rheumatology, Oslo University Hospital, Oslo, Norway; ${ }^{14}$ Neurology; ${ }^{15}$ Ghent University Hospital, Ghent, Belgium;

${ }^{16}$ Rheumatology, University Hospital Zurich, Zurich, Switzerland;

${ }^{17}$ Rheumatology, Arcispedale S. Maria Nuova, Reggio Emilia, Italy; ${ }^{18}$ Royal National Hospital for Rheumatic Diseases: ${ }^{19}$ Pharmacy and Pharmacology, University of Bath, Bath; ${ }^{20}$ Rheumatology, Salford Royal NHS Foundation Trust, Salford, United Kingdom; ${ }^{21}$ ZiteLab ApS, Frederiksberg, Denmark; ${ }^{22}$ Karolinska University Hospital, Karolinska Institutet, Stockholm, Sweden; ${ }^{23} \mathrm{NIHR}$ Manchester Musculoskeletal Biomedical Research Unit, Central Manchester University Hospitals NHS Foundation Trust, Manchester Academic Health Science Centre, Manchester, United Kingdom

Background: The idiopathic inflammatory myopathies (IIM) represent a rare and heterogeneous group of multisystem autoimmune diseases. The rarity of IIM has

Abstract SAT0372 - Table 2

\begin{tabular}{|c|c|c|c|c|c|c|c|}
\hline \multirow[t]{2}{*}{ Clinical Association } & \multicolumn{2}{|c|}{ Whole group $(\mathrm{N}=451)$} & \multirow{2}{*}{$\begin{array}{l}\text { BICD2 alone }(\mathrm{N}=29) \\
\text { Mean (sd) or } \mathrm{N}(\%)\end{array}$} & \multirow{2}{*}{$\begin{array}{c}\text { Overlapping BICD2 }(\mathrm{N}=107) \\
\text { Mean }(\mathrm{sd}) \text { or } \mathrm{N}(\%)\end{array}$} & \multirow{2}{*}{$\begin{array}{c}\text { Negative BICD2 }(\mathrm{N}=315) \\
\text { Mean }(\mathrm{sd}) \text { or } \mathrm{N}(\%)\end{array}$} & \multirow{2}{*}{$\begin{array}{c}\text { BICD2 alone vs Negative } \\
\text { P Values }\end{array}$} & \multirow{2}{*}{$\begin{array}{c}\text { Overlapping vs Negative } \\
\text { P Values }\end{array}$} \\
\hline & Mean (sd) or N (\%) & $\mathrm{NA}$ & & & & & \\
\hline $\begin{array}{l}\text { Disease duration since the } \\
\text { onset of RP, years }\end{array}$ & $15.4(12.9 \%)$ & 18 & $15.6(13.7 \%)$ & $17.5(12.6 \%)$ & $14.6(12.9 \%)$ & 0.728 & 0.013 \\
\hline Diffuse cutaneous disease & $138.0(30.7 \%)$ & 1 & $9.0(31.0 \%)$ & $23.0(21.5 \%)$ & $106.0(33.8 \%)$ & 0.766 & 0.018 \\
\hline Calcinosis & $104.0(23.2 \%)$ & 2 & $8.0(27.6 \%)$ & $33.0(31.1 \%)$ & $63.0(20.1 \%)$ & 0.339 & 0.019 \\
\hline Inflammatory myositis & $43.0(9.6 \%)$ & 4 & $7.0(24.1 \%)$ & $4.0(3.8 \%)$ & $32.0(10.3 \%)$ & 0.003 & 0.040 \\
\hline GERD/reflux & $285.0(69.7 \%)$ & 42 & $16.0(59.3 \%)$ & $79.0(81.4 \%)$ & $190.0(66.7 \%)$ & 0.437 & 0.006 \\
\hline Antibiotics for bacterial growth & $47.0(10.5 \%)$ & 5 & $0.0(0.0 \%)$ & $8.0(7.6 \%)$ & $39.0(12.5 \%)$ & 0.043 & 0.160 \\
\hline Interstitial lung disease & $123.0(27.8 \%)$ & 9 & $13.0(46.4 \%)$ & $17.0(16.5 \%)$ & $93.0(29.9 \%)$ & 0.071 & 0.008 \\
\hline FVC\% predicted & 93.5 (19.5\%) & 61 & $96.0(20.8 \%)$ & $98.3(19.7 \%)$ & $91.7(19.1 \%)$ & 0.304 & 0.004 \\
\hline
\end{tabular}


hampered research efforts, resulting in remarkably limited therapeutic evidence. The EuroMyositis Registry was created to pool resources and expertise across the international IIM research community.

Objectives: To describe the phenotypic characteristics of different IIM subtypes. Associations with malignancy, interstitial lung disease (ILD), cardiac involvement and dysphagia were assessed.

Methods: Pooled data from the EuroMyositis Registry (from Belgium, China, Czech Republic, Hungary, Italy, Mexico, Norway, Sweden, Switzerland, UK, Vietnam) were obtained. Associations were assessed using logistic regression and multinomial logistic regression with polymyositis (PM) as the reference group. Results: Data regarding 3,196 patients were analysed. The UK was the largest contributor $(n=1,307)$. The most common diagnoses were dermatomyositis (34\%), PM (32\%) and connective tissue disease (CTD)-overlap myositis (15\%). In those with anti-synthetase syndrome $(7 \%), 85 \%$ had muscle weakness, $86 \%$ ILD and $58 \%$ arthritis. Overall, $43 \%$ had a myositis specific antibody, most commonly anti-Jo1 autoantibodies (20\%). Glucocorticoid usage was noted in $98 \%$. Most commonly used disease modifying agents were methotrexate $(71 \%)$ and azathioprine (50\%)

Malignancy occurred in $9 \%$ and was associated with a diagnosis of dermatomyositis (Relative Risk Ratio [RRR] 1.68, 95\% Cl 1.09-2.56, $\mathrm{p}=0.018$ ). Cardiac involvement occurred in $9 \%$, most commonly in those with CTD-overlap myositis (13\%), and was associated with a higher Health Assessment Questionnaire disability index (1 versus 0.75 , OR $1.40,95 \% \mathrm{Cl} 1.03-1.91, \mathrm{p}=0.031$ ). Dysphagia occurred in $39 \%$ and was associated with a diagnosis of CTD-overlap myositis (RRR 2.25, 95\% Cl 1.61-3.15, p<0.001).

Conclusions: This large international cohort demonstrates the heterogeneity of IIM and the burden of associated malignancy, ILD, cardiac and gastrointestinal involvement. The EuroMyositis Registry facilitates international collaborative research outputs, underlining the benefits of harmonised data collection methodology between centres.

Acknowledgements: This work was supported by researchers at the National Institute for Health Research (NIHR) Biomedical Research Unit. The views expressed are those of the authors and not necessarily those of the UK National Health Service, the NIHR or the UK Department of Health

Disclosure of Interest: J. Lilleker: None declared, J. Vencovsky: None declared, G. Wang: None declared, L. Wedderburn Grant/research support from: The UK JDM Cohort and Biomarker study is supported by grants from the NIHR and Myositis UK, L. Diederichsen: None declared, J. Schmidt: None declared, P. Jordan: None declared, O. Benveniste: None declared, M. G. Danieli: None declared, K. Dankó: None declared, N. T. P. Thuy: None declared, M. VazquezDel Mercado: None declared, $\varnothing$. Molberg: None declared, B. De Paepe: None declared, J. De Bleecker: None declared, B. Maurer Grant/research support from: AbbVie, Protagen, EMDO, Novartis, Roche, Actelion, N. Pipitone Speakers bureau: GRAPPA Workshop, Alfa-Wassermann, N. McHugh: None declared, Z. Betteridge: None declared, P. New: None declared, R. Cooper: None declared, W. Ollier: None declared, J. Lamb Grant/research support from: Medlmmune, N. S. Krogh: None declared, I. Lundberg Grant/research support from: Astra-Zeneca, Bristol-Myers Squibb, Consultant for: Bristol-Myers Squibb, Idera, H. Chinoy Grant/research support from: Medlmmune, Novartis

DOI: 10.1136/annrheumdis-2017-eular.2742

\section{SAT0375 METABOLOMICS OF SERA REVEALS POTENTIAL BIOMARKERS OF SKIN FIBROSIS IN SYSTEMIC SCLEROSIS THAT CORRELATE WITH PRO-FIBROTIC GENE EXPRESSION IN SKIN BIOPSIES}

S. Ahmed ${ }^{1}$, M.K. Rai ${ }^{1}$, D. Dubey ${ }^{2}$, A. Rawat ${ }^{2}$, D.P. Misra ${ }^{1}$, D. Kumar ${ }^{2}$, V. Agarwal ${ }^{1} .{ }^{1}$ Clinical Immunology, Sanjay Gandhi Post Graduate Institute of Medical Sciences; ${ }^{2}$ Centre for BioMedical Research, Lucknow, India

Background: There is an unmet need for biomarkers in Systemic Sclerosis (SSc). Despite its shortcomings, the modified Rodnan skin score (mRSS) has remained the standard disease assessment tool for SSc. Expression of certain genes, cartilage oligomeric matrix protein (COMP), thrombospondin-1 (THS1), interferon-induced 44 (IFI44) and sialoadhesin (SIG1), and, more recently, Tenascin-C (TNSC), have been shown to correlate with skin fibrosis. However, assessment of the expression of these genes requires a skin biopsy. Hence, we used an open-ended approach to identify a serum-based biomarker of SSc.

Objectives: To identify small molecules in serum that correlate with mRSS and profibrotic genes that are upregulated in skin of SSc patients

Methods: We obtained serum and skin biopsies from 25 consenting adult patients with SSc and serum from 25 age- and sex-similar controls. mRNA levels of five genes: COMP, THS1, IFI44, SIG1, and TNSC were estimated as fold-change relative to Glyceraldehyde 3-phosphate dehydrogenase (GAPDH), a housekeeping gene. $\mathrm{H}^{1} \mathrm{NMR}$ (Nuclear Magnetic Resonance) based metabolomics studies were performed on the sera using standard protocols. Principal component analysis (PCoA) and Partial Least Squares Discriminant Analysis (PLSDA) were used to delineate metabolites that were different between patients and healthy controls. Then spearman correlations $(\rho)$ of these metabolites with mRSS and the fold-expression of the five pro-fibrotic genes were estimated.

Results: $\mathrm{H}^{1} \mathrm{NMR}$ based metabolomics identified 126 peaks that were different between patients and controls. Out of these, the levels of glycine had the best correlation with pro-fibrotic gene expression $(\rho=0.5, p<0.05$ for IFI44; $\rho=0.44$,
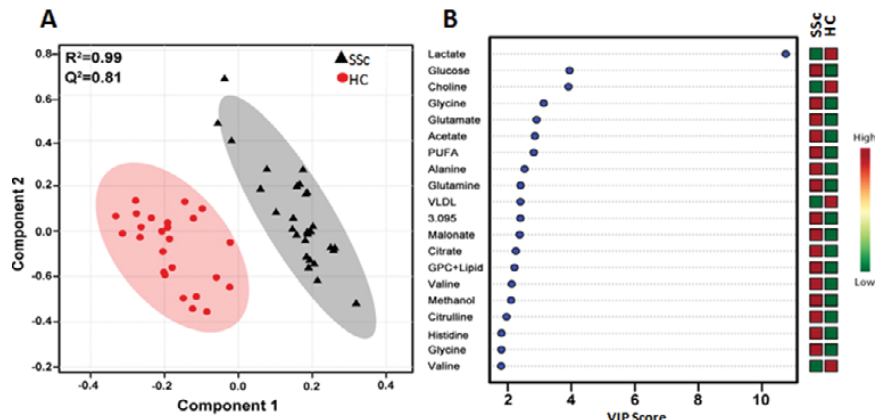

Figure 1: $\mathrm{H}^{1}$-NMR Metabolomics data (A) PCOA plot showing metabolomics can clearly distinguish between patients of systemic sclerosis and healthy controls (B) Plot showing 20 representative metabolites that were significantly different between patients and controls; SSc: Systemic Sclerosis, HC: Healthy Controls, PUFA: Poly Unsaturated Fatty Acids; VLDL: Very Low Density Lipoproteins, GPC: Glycerophosphocholine, VIP: Variable Importance in Projection Score

$\mathrm{p}<0.05$ for TNSC). Choline inversely correlated with SIG1 $(\rho=-0.41 ; \mathrm{p}=0.05)$ while Glycerophosphocholine correlated with $\operatorname{mRSS}(\rho=0.50, p<0.05)$.

Conclusions: $\mathrm{H}^{1} \mathrm{NMR}$ based metabolomics identified glycine, choline and their metabolites as potential biomarkers for skin fibrosis in SSc. These findings require validation in a larger cohort

References:

[1] Farina G, Lafyatis D, Lemaire R, Lafyatis R. A four-gene biomarker predicts skin disease in patients with diffuse cutaneous systemic sclerosis. Arthritis Rheum. 2010 Feb;62(2):580-8.

[2] Bhattacharyya S, Wang W, Morales-Nebreda L, Feng G, Wu M, Zhou X, Lafyatis R, Lee J, Hinchcliff M, Feghali-Bostwick C, Lakota K, Budinger GR, Raparia K, Tamaki Z, Varga J.Tenascin-C drives persistence of organ fibrosis. Nat Commun. 2016 Jun 3:7:11703.

Disclosure of Interest: None declared

DOI: 10.1136/annrheumdis-2017-eular.3715

\section{SAT0376 ANTI-NT5C1A AUTOANTIBODIES ARE FREQUENT IN JUVENILE MYOSITIS AND ASSOCIATED WITH INCREASED ILLNESS SEVERITY}

R. Yeker ${ }^{1}$, I. Pinal-Fernandez ${ }^{1}$, T. Kishi ${ }^{2}$, I.N. Targoff ${ }^{3}$, F.W. Miller ${ }^{2}$, L.G. Rider $^{2}$ A. Mammen ${ }^{1} .{ }^{1}$ Muscle Disease Unit, National Institute of Arthritis and Musculoskeletal and Skin Diseases (NIAMS)/National Institutes of Health (NIH); ${ }^{2}$ Environmental Autoimmunity Group, National Institute of Environmental Health Sciences (NIEHS)/National Institutes of Health $(\mathrm{NIH})$, Bethesda; ${ }^{3}$ University of Oklahoma Health Sciences Center, Oklahoma City, United States

Background: Autoantibodies (Abs) against 5'-Nucleotidase, Cytosolic 1A (NT5C1A) have been portrayed as a potential diagnostic aide in distinguishing inclusion body myositis (IBM) and polymyositis (PM) in adults. However, $4-21 \%$ of dermatomyositis (DM) patients also have Abs to NT5C1A.

Objectives: The prevalence and clinical features of anti-NT5C1A Abs in juvenileonset myositis (IIM) patients is unknown, so we sought to examine this in a large cohort.

Methods: We screened 384 juvenile IIM patients meeting probable or definite Bohan and Peter criteria for anti-NT5C1A Abs by immunoblotting for the full length NT5C1A protein in transfected and non-transfected lysates. Clinical characteristics and HLA typing were compared between juvenile IIM patients who were anti-NT5C1A positive $(A b+)$ and those who were anti-NT5C1A negative $(\mathrm{Ab}-)$

Results: In this cohort, $29 \%$ (103) of juvenile DM, $15 \%$ (3) of juvenile PM, and $37 \%(15)$ of juvenile overlap myositis patients tested positive for anti-NT5C1A Abs. Compared with anti-NT5C1A Ab-patients, anti-NT5C1A Ab+ juvenile IIM patients showed a similar distribution of race, gender, and association with myositis-specific Abs. However, NT5C1A Ab was associated with anti-p155/140 Abs $(p=0.05)$. The only observed clinical difference was an increased frequency of $\mathrm{V}$ - or shawl-sign rashes ever present $(44 \%$ vs. $26 \%, p=0.002)$. Disease severity was increased in anti-NT5C1A Ab+ patients, based on more frequent hospitalizations $(p=0.02)$, more medications used $(p<0.001)$, and more treatment trials per year $(p<0.001)$. Additionally, pulse steroids $(p<0.001)$ and intravenous immunoglobulin therapy $(p=0.008)$ were prescribed more frequently in anti-NT5C1A Ab+than Ab- patients. The HLA alleles DRB1*07 (20\% vs. $9 \%, p<0.05)$ and DQA $1^{*} 0201(21 \%$ vs. $7 \%$, $p<0.01$ ) were present more frequently in anti-NT5C1A Ab- compared to Abpatients.

Conclusions: Anti-NT5C1A Abs are commonly present in juvenile DM and juvenile overlap myositis patients, and are present more frequently in patients with anti-p155/140 Abs, but are also seen in association with other myositis specific Abs. Consistent with data in adult IIM patients, anti-NT5C1A Abs have few distinguishing clinical features in juvenile myositis, but are associated with increased illness severity marked by increased hospitalizations and receipt of additional therapy.

Disclosure of Interest: R. Yeker: None declared, I. Pinal-Fernandez Grant/ research support from: The Myositis Association, T. Kishi Grant/research support 